\title{
EMPLOI DE LA DL-MÉTHIONINE ET DE LA L-LYSINE HCI POUR RÉDUIRE L'APPORT ALIMENTAIRE DE PROTÉINES CHEZ LE PINTADEAU DE CHAIR
}

\author{
B. LECLERCQ, M. LARBIER et J. C. BLUM \\ avec la collaboration technique de Solange Gulllaumin et R. Bonsergent \\ Station de Recherches avicoles, \\ Centre de Recherches de Tours, I. N. R. A., \\ B.P. 1 Nousilly, 37380 Monnaie
}

\section{RÉSUMÉ}

Dans un régime ì base de maïs et de soja titrant $16,7 \mathrm{p}$. roo de matières azotées (MAT : $\mathrm{N} \times 6,25)$ la méthionine est l'acide aminé limitant la croissance du Pintadeau entre 4 et 8 semaines d'âge. Il en est de même entre 8 et I 2 semaines avec un régime titrant r 2,7 p. Ioo de MAT. Avec ces deux régimes les performances sont également améliorées par le supplément de DL-méthionine synthétique ou par son équivalent ajouté avec $2 \mathrm{p}$. Ioo de protéines de soja (2 régimes témoins). Ainsi, la DL-méthionine peut être utilisée pour économiser des protéines ; il en est tout autrement pour la L-lysine qui n'est pas un facteur limitant des deux régimes pauvres en protéines.

Chez le Pintadeau âgé de 4 à 8 semaines, les besoins sont inférieurs à o, $88 \mathrm{p}$. Ioo dans le cas de la lysine, et se situent entre 0,62 et 0,76 p. Ioo pour les acides aminés soufrés. Aux âges de 8 à I2 semaines, ces besoins, inférieurs à o,53 p. Ioo pour la lysine, sont compris entre o,45 et o,55 p. Ioo pour les acides aminés soufrés. Des analyses corporelles effectuées à l'âge de $\mathrm{I} 2$ semaines montrent que l'état d'engraissement dépend indirectement de la nature des régimes. En accélérant le développement pondéral, les acides aminés limitants favorisent l'engraissement qui apparaît tardivement chez les animaux lourds.

\section{INTRODUCTION}

Le Pintadeau a la réputation d'un animal exigeant des régimes très riches en protéines. Deux d'entre nous ont eu l'occasion de préciser l'importance du besoin en constituants azotés au cours des différentes périodes de sa croissance (BLUM, Guillaume, LECLERCQ, I975). Ce besoin n'est pas aussi élevé qu'on le prétendait. Mais il apparaît cependant anormalement élevé, compte tenu d'un développement pondéral relativement lent. Cela peut tenir à une exigence particulière envers cer- 


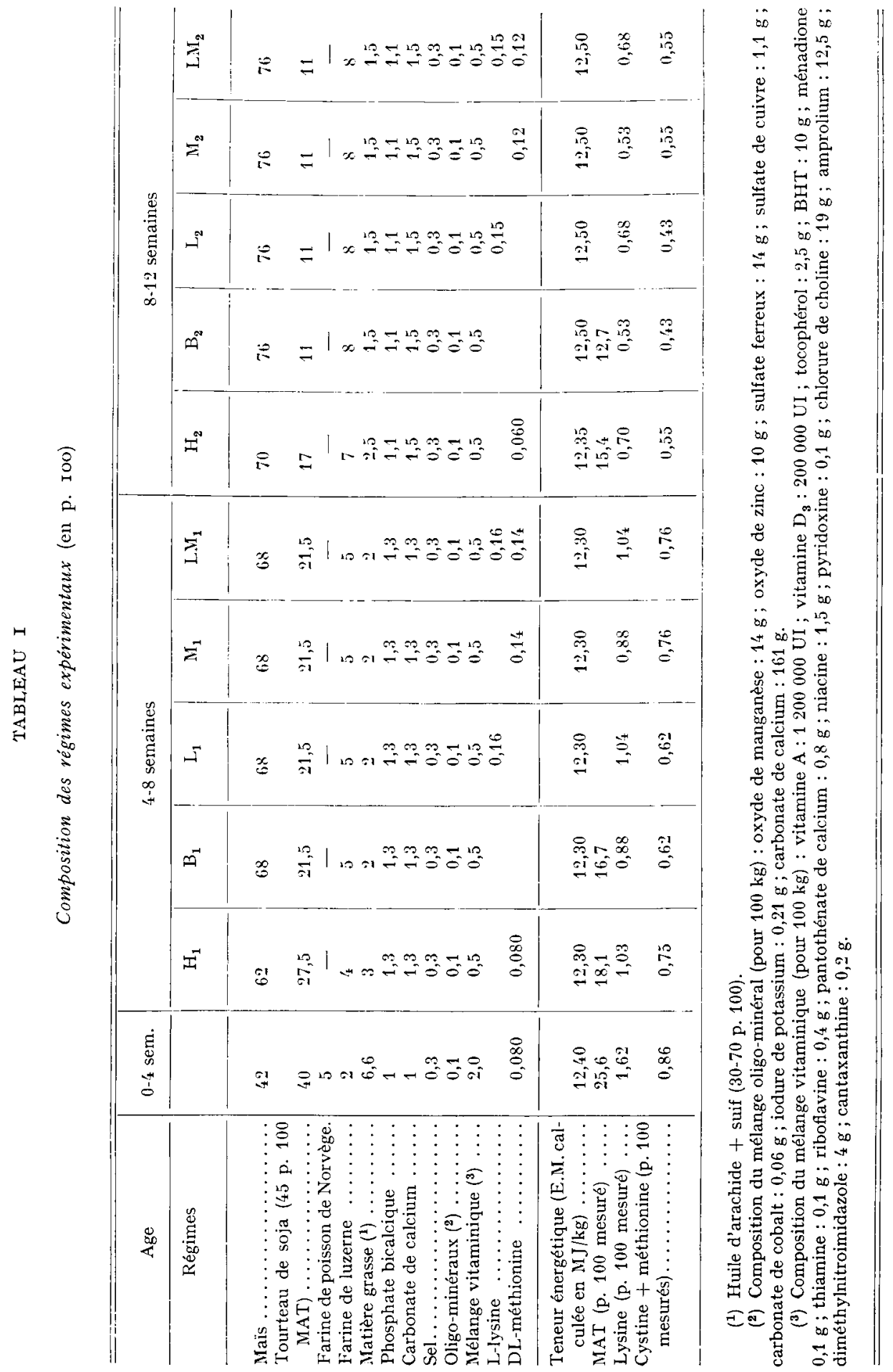


tains acides aminés indispensables. Dans ce cas, il doit être possible d'abaisser la concentration en protéines des régimes par l'addition d'acides aminés de synthèse sans réduire les performances de croissance. C'est l'objet de ce travail qui porte sur l'utilisation de lysine et de méthionine chez le Pintadeau entre 4 et 12 semaines.

\section{MATÉRIEL ET MÉTHODES}

Les pintadeaux sont élevés dans 22 cases de $6 \mathrm{~m}^{2}$ sur litières de copeaux de bois. On place 50 animaux par case. Le chauffage est assuré par air pulsé sous une hotte placée dans le coin de la case.

La composition des régimes est rapportée dans le tableau $\mathbf{r}$, le schéma expérimental fait l'objet du tableau 2. Pendant les 4 premières semaines de vie, les animaux disposent tous du même aliment. Les régimes distribués au cours des deux périodes suivantes ( 4 - 8 semaines et 8 - I 2 semaines) définissent I I lots ( 2 répétitions par lot). Ces régimes sont choisis pour satisfaire le besoin en matières azotées (régimes $\mathrm{H}_{1}$ et $\mathrm{H}_{2}$ ) ou au contraire pour apporter une quantité légèrement insuffisante de protéines (régimes $\mathrm{B}_{1}$ et $\mathrm{B}_{2}$ ). Les régimes $\mathrm{B}$ peuvent être supplémentés soit par de la lysine $\left(L_{1}\right.$ et $\left.L_{2}\right)$, soit par de la méthionine $\left(M_{1}\right.$ et $\left.M_{2}\right)$, soit par les 2 acides aminés simultanément $\left(\mathrm{LM}_{1}\right.$ et $\left.\mathrm{LM}_{2}\right)$. Ces supplémentations sont calculées de façon à effacer les différences entre les régimes $B$ et $H$ pour les acides aminés étudiés (lysine et acides aminés soufrés).

TABLEAU 2

Schéma expérimental

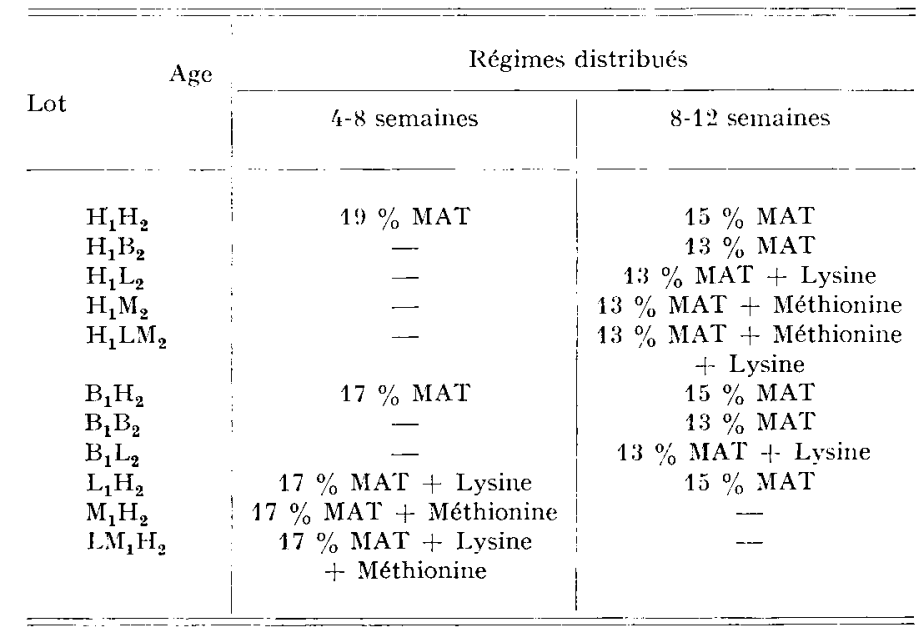

Les animaux et les quantités d'aliment consommées sont pesés aux stades de 4, 8 et I 2 semaines. De plus, à $I_{2}$ semaines $I_{5}$ animaux de chacun des lots $H_{1} H_{2}, B_{1} B_{2}$ et $H_{1} M_{2}$ sont sacrifiés et plumés en vue d'analyse corporelle. Cette dernière est effectuée selon les méthodes utilisées habituellement dans notre laboratoire (Leclerce, Delpech et Blum, i969).

Enfin la composition des aliments en acides aminés (tabl. 3) est déterminée par chromatographie sur résine échangeuse de cations selon la méthode de Moore. Spackman et Stein (i958) après oxydation à l'acide performique et hydrolyse à l'acide chlorhydrique $6 \mathrm{~N}$ pendant 24 heurcs selon la méthode décrite par Pion et Fauconneau (Ig66). 
TABLEAU 3

Composition en acides aminés des régimes cxpérimentaux

(Analyse chromatographique)

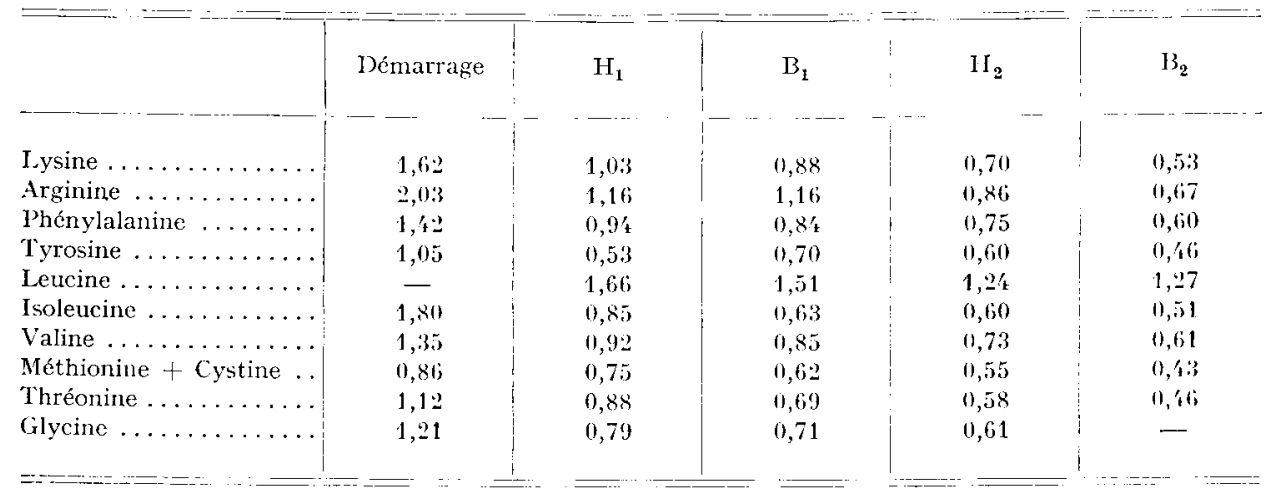

\section{RÉSUL'TA'TS E'T DISCUSSION}

Les performances zootechniques font l'objet du tableau 4. A l'âge de 4 semaines la croissance que permet le régime distribué à l'ensemble des animaux est correcte. Les différents régimes $\left(\mathrm{H}_{1}, \mathrm{~B}_{1}, \mathrm{M}_{1}, \mathrm{~L}_{1}\right.$ et $\left.\mathrm{L} \mathrm{M}_{1}\right)$ distribués au cours de la période suivante influencent les résultats obtenus à l'âge de 8 semaines. La supplémentation en lysine du régime $B_{1}$ est inefficace. Au contraire, l'addition de méthionine à ce régime permet d'obtenir une croissance pratiquement identique à celle réalisée avec le régime $\mathrm{H}_{1}$ riche en protéines. La supplémentation simultanée en lysine et méthionine n'apporte aucun avantage supplémentaire. La valeur de l'indice de consommation dépend de la croissance. Elle est plus faible avec les régimes $H_{1}, M_{1}$ et $L M_{1}$. Fintre les âges de 4 et 8 semaines, la méthionine s'est avérée le seul acide aminé limitant du régime $B_{1}$ pauvre en protéines.

A l'âge de $\mathrm{I} 2$ semaines, la comparaison des lots $\mathrm{H}_{1} \mathrm{H}_{2}, \mathrm{H}_{1} \mathrm{~B}_{2}, \mathrm{H}_{1} \mathrm{~L}_{2}, \mathrm{H}_{1} \mathrm{M}_{2}$ et $\mathrm{H}_{1} \mathrm{~L}_{1} \mathrm{M}_{2}$ indique que la méthionine demeure, durant la période de finition, l'acide aminé limitant. La supplémentation en lysine seule ou jointe à la méthionine n'exerce aucun effet bénéfique. Si l'on met en parallèle les résultats des lots $\mathrm{B}_{1} \mathrm{H}_{2}, \mathrm{M}_{1} \mathrm{H}_{2}, \mathrm{~L}_{1} \mathrm{H}_{2}$, $\mathrm{L}_{1} \mathrm{M}_{1} \mathrm{H}_{2}$ et $\mathrm{H}_{1} \mathrm{H}_{2}$, on constate que le régime $\mathrm{H}_{2}$ permet aux lots retardés entre l'âge de 4 et 8 semaines de combler la majeure partie de leur retard de croissance.

Nous présentons dans le tableau 5 les résultats d'analyse de la composition corporelle. Ires pintadeaux recevant les régimes riches en protéines sont toujours plus gras que ceux nourris avec des régimes moins bien pourvus en constituants azotés. Nous confirmons donc notre première observation (BLUM, GUILLAUME, LECLIRCQ, 1975). Le Pintadeau réagit donc à l'inverse du Poulet, il engraisse lorsque l'apport alimentaire d'acides aminés est suffisant pour couvrir les besoins. En fait, les différences d'état d'engraissement sont liées aux différences de poids vif. I a corrélation entre ces deux caractéristiques est de $+0,622$ et l'analyse de covariance révèle que 





l'effet des régimes n'est plus significatif lorsque le poids des animaux est pris en considération. Finalement, le développement pondéral détermine dans une large mesure la composition corporelle.

TABL,FAU 5

Composition chimique de la carcasse de Pintadeau plumé et éviscéré (p. 100 de produit frais)

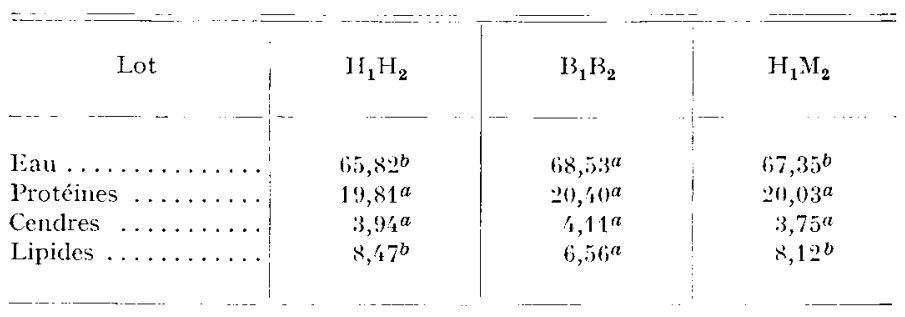

Les moyennes suivies d'une même lettre ne sont pas significativement différentes au seuil de signification de 5 p. 100.

\section{CONCLUSION}

Nous avions précédemment montré qu'il est possible d'abaisser le taux de matières protéiques généralement recommandé dans les régimes destinés au Pintadeau sans modifier sa croissance. En jouant sur l'équilibre des acides aminés de la ration, en particulier en augmentant la teneur en acides aminés soufrés, il est possible de réduire encore davantage l'apport de protéines nécessaires. Certes, cette expérimentation est insuffisante pour permettre, à elle seule, de fixer la valeur exacte du besoin en lysine et méthionine. On peut cependant affirmer qu'entre 4 et 8 semaines le besoin en lysine est inférieur à $0,88 \mathrm{p}$. Ioo du régime. De même entre 8 et I 2 semaines, il est inférieur à 0,53 p. Ioo. Pour ce qui concerne les acides aminés soufrés, le besoin se situe entre 0,62 et 0,76 p. Ioo durant la période de 4 à 8 semaines, entre 0,43 et 0,55 p. Ioo pendant les 4 semaines suivantes ( 8 et I 2 semaines). La détermination exacte du besoin exige donc de nouvelles expérimentations.

D'ores et déjà on peut remarquer que le rapport besoin en acides aminés soufrés est nettement plus faible chez le Pintadeau que chez le Poulet. Pour ce dernier, Combs (I96I) propose la valeur de I,47. Nos résultats suggèrent qu'elle est tout au plus légèrement supérieure à $I$ pour le Pintadeau. Ce dernier aurait donc un besoin important en acides aminés soufrés dont il convient de préciser la cause et de savoir s'il persiste à l'état adulte, en particulier au cours de la saison de ponte. 


\title{
SUMMARY
}

\author{
REDUCTION OF THE DIETARY PROTEIN SUPPLY \\ IN THE GUINEA FOWL BY MEANS OF DL-METHIONINE \\ AND L_LYSINE HCL SUPPLEMENTATION
}

Growing-finishing guinea fowls were reared from the age of 4 to 12 weeks according to an experimental schedule including two successive periods of four weeks. The animals were fed isoenergetic diets (table I) with different protein levels : I8. I and I6.7 p. Ioo during the first period and $x_{5.4}$ to 12.7 p. Ioo during the second one. The amino acid content of these diets was determined by chromatography (table 3 ). The diets containing $x 6.7$ and 12.7 p. Ioo protein were offered either without or with addition of one or both of the two synthetic amino acid supplements, DL-methionine and L-lysine $\mathrm{HCl}$ (table 2).

Whatever the period of rearing, the diets with the lowest protein content were improved very markedly by the addition of DL-methionine. The performances of the animals were comparable to those obtained with the higher protein diets (table 4). The requirements for sulphur amino acids seemed to be located between 0.62 and $0.76 \mathrm{p}$. I oo of the diet in the 4 to 8 weeks old guinea fowls and ranged between 0.45 and $0.55 \mathrm{p}$. 100 in the birds aged 8 to $\mathrm{I} 2$ weeks.

On the other hand, none of the diets used seemed to be lysine deficient. The addition of L lysine $\mathrm{HCl}$ alone or combined with DL-methionine did not improve the animals' performances (table 4 ). Thus, lysine requirements are lower than 0.88 and $0.53 \mathrm{p}$. Ioo, respectively for birds between the ages of $4-8$ weeks and 8-12 weeks.

The body analyses made at the age of 12 weeks showed that the fatness of the animals depended indirectly on the nature of the diets (table 5). The live weight is determinative; the heaviest guinea fowls are fatty. By accelerating the weight gain, the limiting amino acids favoured the fattening.

\section{RÉFÉRENCES BIBLIOGRAPHIQUES}

Blum J. C., Guillaume J., Leclerce B., 1975. Studies of the energy and protein requirements of the growing guinea fowl. Brit. Poult. Sci. (sous presse).

Combs G. F., I96I. Maryland broiler nutrition studies. Proc. Maryl. Nutr. Conf., 5r-64.

Leclerco B., Delpech P., Blum J. C., 1969. Influence du régime maternel sur la croissance de jeune poussin. Effets d'une déficience en acide linoléique. Ann. Biol. anim. Bioch. Biophys., 9, I9I-204.

Moore S., Spackman D. H., Stein H. W., I958. Chromatography of amino acids on sulfonated polystyrene resins. Analyt. Chem., 30, iा 85-I I90.

Pion R., Fauconneau G., 1966. Les acides aminés des protéines alimentaires : méthodes de dosage et résultats obtenus, in $A$ mino acides, peptides, protéines, cahier no $6, \mathrm{I}_{59}-\mathrm{I} 75, \mathrm{~A}$. E. C. Société de Chimie organique et biologique, Commentry. 